\title{
Structure and Dynamics of a Dihydrogen/Hydride Ansa Molybdenocene
}

\section{Complex}

\author{
Vincent Pons, ${ }^{\&}$ Stephen L. J. Conway, Malcolm L. H. Green, \\ Jennifer C. Green,, Benjamin J. Herbert" and D. Michael Heinekey ${ }^{\text {\& }}$ \\ \&Department of Chemistry, University of Washington, Box 351700, Seattle, WA \\ $98195-1700$ \\ "Inorganic Chemistry Laboratory, South Parks Road, Oxford, OX1 3QR, UK
}

\section{Optimised Geometries}

Coordinates
Mo $\quad 0.029530000 \quad 0.007012000-0.000558000$
C $\quad 0.264483000-0.690448000-2.199457000$
C $\quad-0.183148000 \quad 0.647073000 \quad-2.238751000$
C $\quad-1.433982000 \quad 0.732579000-1.582581000$
C $-1.780971000-0.582109000-1.129387000$
C $-0.708995000-1.461692000-1.513693000$
$\begin{array}{llll}\text { C } & -0.187220000 & 0.649991000 & 2.236644000\end{array}$
$\begin{array}{llll}\text { C } & 0.262398000 & -0.687031000 & 2.200468000\end{array}$
$\begin{array}{llll}\text { C } & -0.708665000 & -1.461591000 & 1.514769000\end{array}$
$\begin{array}{llll}\text { C } & -1.781359000 & -0.584727000 & 1.128433000\end{array}$
$\begin{array}{llll}\text { C } & -1.438122000 & 0.731360000 & 1.580194000\end{array}$
$\begin{array}{llll}\text { C } & -2.757006000 & -0.882765000 & 0.000457000\end{array}$
$\mathrm{H} \quad-0.661441000 \quad-2.538590000 \quad 1.357660000$
H $\quad 1.185661000 \quad-1.064484000 \quad 2.638996000$
$\mathrm{H} \quad-2.037317000 \quad 1.634491000 \quad 1.462809000$
$\mathrm{H} \quad 0.348681000 \quad 1.487837000 \quad 2.684889000$
$\begin{array}{llll}\text { C } & -3.939867000 & 0.052059000 & 0.003575000\end{array}$
$\begin{array}{llll}\text { C } & -3.211646000 & -2.320720000 & -0.011766000\end{array}$
$\mathrm{H} \quad-0.663995000 \quad-2.538397000-1.353806000$
$\begin{array}{lllll}\mathrm{H} & 1.188662000 & -1.070291000 & -2.634049000\end{array}$
$\mathrm{H} \quad 0.355916000 \quad 1.483567000 \quad-2.685556000$
$\mathrm{H} \quad-2.029461000 \quad 1.638162000-1.466424000$
$\mathrm{H} \quad 1.460206000 \quad-0.889354000 \quad 0.000005000$
$\mathrm{H} \quad 0.809448000 \quad 1.593202000-0.001673000$
$\mathrm{H} \quad 1.546909000 \quad 0.940640000 \quad-0.003114000$
$\begin{array}{llll}\mathrm{H} & -4.530808000 & -0.099788000 & 0.921146000\end{array}$
$\mathrm{H} \quad-3.645774000 \quad 1.112522000 \quad-0.043414000$
$\mathrm{H} \quad-4.591738000 \quad-0.163865000-0.857636000$
$\mathrm{H} \quad-3.843599000 \quad-2.522265000 \quad 0.867907000$
H $\quad-3.814466000 \quad-2.513308000-0.913752000$ 


\section{Frequencies}

\begin{tabular}{|c|c|c|}
\hline $\begin{array}{l}\text { Frequency } \\
\mathrm{cm}-1\end{array}$ & $\begin{array}{l}\text { Dipole Strength } \\
1 \mathrm{e}-40 \text { esu } 2 \mathrm{~cm} 2\end{array}$ & $\begin{array}{l}\text { Absorption Intensity (degeneracy not counted) } \\
\mathrm{km} / \mathrm{mole}\end{array}$ \\
\hline --------- & 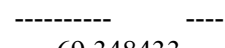 & $-0_{990325}$ \\
\hline $\begin{array}{c}56.972188 \\
180.591895\end{array}$ & $\begin{array}{r}69.348433 \\
3.677179\end{array}$ & $\begin{array}{l}0.990325 \\
0.166453\end{array}$ \\
\hline 188.118846 & 4.464643 & 0.210522 \\
\hline 217.922065 & 42.634153 & 2.328825 \\
\hline 237.025139 & 11.178017 & 0.664106 \\
\hline 240.842726 & 127.107996 & 7.673341 \\
\hline 269.465212 & 48.585239 & 3.281596 \\
\hline 272.122633 & 95.572112 & 6.518894 \\
\hline 300.159088 & 20.511545 & 1.543220 \\
\hline 302.952048 & 22.619891 & 1.717681 \\
\hline 357.977034 & 6.879479 & 0.617289 \\
\hline 396.503114 & 35.426102 & 3.520854 \\
\hline 406.918036 & 364.415583 & 37.169092 \\
\hline 419.379379 & 186.627871 & 19.618312 \\
\hline 429.988239 & 158.706571 & 17.105255 \\
\hline 483.421654 & 215.854659 & 26.155655 \\
\hline 504.690363 & 87.778970 & 11.104361 \\
\hline 537.442224 & 178.439477 & 24.038136 \\
\hline 550.651271 & 44.293873 & 6.113619 \\
\hline 600.179911 & 0.045021 & 0.006773 \\
\hline 606.496552 & 54.007020 & 8.210255 \\
\hline 610.796489 & 5.469142 & 0.837325 \\
\hline 639.905549 & 344.889546 & 55.318956 \\
\hline 720.756167 & 20.491459 & 3.702025 \\
\hline 735.443808 & 127.373403 & 23.480445 \\
\hline 739.549899 & 25.337948 & 4.696961 \\
\hline 797.701619 & 99.768656 & 19.948611 \\
\hline 811.889104 & 9.797657 & 1.993871 \\
\hline 815.648662 & 72.018349 & 14.723951 \\
\hline 819.388356 & 2.972285 & 0.610462 \\
\hline 840.071531 & 47.952353 & 10.097277 \\
\hline 840.636849 & 8.169565 & 1.721414 \\
\hline 855.497878 & 55.371285 & 11.873578 \\
\hline 857.117113 & 58.384087 & 12.543327 \\
\hline 869.137387 & 15.128763 & 3.295869 \\
\hline 880.167861 & 1.311063 & 0.289246 \\
\hline 906.988696 & 1.978477 & 0.449791 \\
\hline 911.110247 & 39.795489 & 9.088304 \\
\hline 918.203468 & 3.408773 & 0.784540 \\
\hline 926.820458 & 20.408579 & 4.741180 \\
\hline 930.350833 & 83.344472 & 19.435764 \\
\hline 967.312381 & 0.143523 & 0.034799 \\
\hline 995.743742 & 550.502788 & 137.399508 \\
\hline 1011.280943 & 15.769121 & 3.997214 \\
\hline 1018.031908 & 0.145709 & 0.037181 \\
\hline 1020.520284 & 16.476217 & 4.214608 \\
\hline 1026.179464 & 56.388394 & 14.504111 \\
\hline 1050.271648 & 1.145281 & 0.301503 \\
\hline 1055.785454 & 33.048003 & 8.745788 \\
\hline 1117.875014 & 3.864656 & 1.082885 \\
\hline 1142.624743 & 22.143543 & 6.342037 \\
\hline 1154.130959 & 3.447428 & 0.997306 \\
\hline 1178.535970 & 0.609768 & 0.180130 \\
\hline 1219.718893 & 0.266521 & 0.081483 \\
\hline 1224.958660 & 3.171251 & 0.973711 \\
\hline 1271.982195 & 100.329856 & 31.988162 \\
\hline 1327.162753 & 0.737024 & 0.245179 \\
\hline 1330.771244 & 39.903291 & 13.310372 \\
\hline 1349.548173 & 68.329735 & 23.114058 \\
\hline
\end{tabular}




$\begin{array}{lcl}1359.879532 & 23.499591 & 8.010116 \\ 1395.095833 & 1.844852 & 0.645125 \\ 1400.391790 & 17.614137 & 6.182854 \\ 1401.180709 & 29.834596 & 10.478337 \\ 1405.021979 & 1.721514 & 0.606278 \\ 1409.370879 & 21.323075 & 7.532744 \\ 1410.536260 & 8.802601 & 3.112242 \\ 1416.719493 & 2.394195 & 0.850201 \\ 1424.332474 & 20.944551 & 7.477570 \\ 1433.636555 & 52.190232 & 18.754538 \\ 1440.996699 & 41.290041 & 14.913733 \\ 1856.573798 & 47.356475 & 22.037872 \\ 1945.655975 & 26.404411 & 12.877175 \\ 2098.148916 & 75.000548 & 39.443806 \\ 2955.342940 & 11.257587 & 8.339332 \\ 2956.789612 & 0.386886 & 0.286735 \\ 3040.477721 & 3.522217 & 2.684331 \\ 3041.581784 & 5.730780 & 4.369093 \\ 3042.570699 & 0.409811 & 0.312537 \\ 3043.984237 & 1.279595 & 0.976322 \\ 3146.206922 & 5.639659 & 4.447523 \\ 3146.734005 & 12.386384 & 9.769732 \\ 3157.277648 & 9.860637 & 7.803615 \\ 3157.435677 & 12.976521 & 10.270010 \\ 3157.644659 & 29.365713 & 23.242448 \\ 3158.377883 & 69.569526 & 55.075849 \\ 3169.025846 & 6.064483 & 4.817233 \\ 3169.296650 & 32.589514 & 25.889212\end{array}$

\section{$\mathbf{1 - C _ { 2 V }}$}

\section{Coordinates}

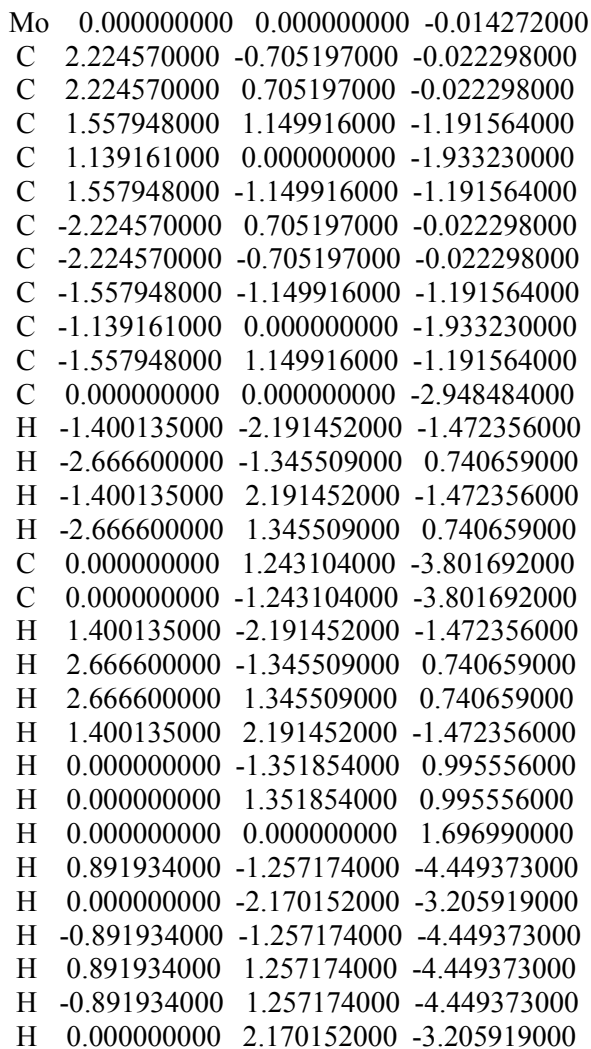

Frequencies 


\begin{tabular}{|c|c|c|}
\hline $\begin{array}{l}\text { Frequency } \\
\mathrm{cm}-1\end{array}$ & $\begin{array}{l}\text { Dipole Strength } \\
1 \mathrm{e}-40 \text { esu } 2 \mathrm{~cm} 2\end{array}$ & $\begin{array}{l}\text { Absorption Intensity (degeneracy not counted) } \\
\mathrm{km} / \text { mole }\end{array}$ \\
\hline ---------- & --------- $\quad$---- & ---- \\
\hline-90.967966 & 6.487595 & -0.147928 \\
\hline 183.877174 & 0.000000 & 0.000000 \\
\hline 188.584553 & 263.192537 & 12.441071 \\
\hline 226.107823 & 1415.109525 & 80.201726 \\
\hline 228.913776 & 24.787190 & 1.422254 \\
\hline 240.692633 & 238.543801 & 14.391597 \\
\hline 243.410165 & 0.000000 & 0.000000 \\
\hline 266.269566 & 41.461874 & 2.767251 \\
\hline 276.314360 & 0.000000 & 0.000000 \\
\hline 302.051781 & 25.365324 & 1.920436 \\
\hline 308.809203 & 5.306770 & 0.410770 \\
\hline 357.628835 & 1.297971 & 0.116352 \\
\hline 392.318698 & 19.824097 & 1.949443 \\
\hline 415.089553 & 0.000000 & 0.000000 \\
\hline 429.824777 & 86.453348 & 9.314324 \\
\hline 434.694733 & 28.219043 & 3.074714 \\
\hline 461.098693 & 913.056239 & 105.528433 \\
\hline 508.925350 & 13.296625 & 1.696186 \\
\hline 518.935274 & 410.307907 & 53.370485 \\
\hline 546.014110 & 13.397026 & 1.833540 \\
\hline 575.413413 & 243.056552 & 35.056244 \\
\hline 601.511090 & 0.000000 & 0.000000 \\
\hline 619.495857 & 5.651964 & 0.877639 \\
\hline 623.424903 & 121.516300 & 18.988767 \\
\hline 735.309048 & 63.132407 & 11.635909 \\
\hline 748.063353 & 0.000000 & 0.000000 \\
\hline 764.054678 & 144.092481 & 27.595853 \\
\hline 790.231540 & 341.655460 & 67.673836 \\
\hline 818.561077 & 0.000000 & 0.000000 \\
\hline 820.642572 & 13.770111 & 2.832498 \\
\hline 825.499842 & 31.571429 & 6.532649 \\
\hline 852.261288 & 0.000000 & 0.000000 \\
\hline 852.771763 & 78.837222 & 16.851641 \\
\hline 861.935163 & 0.982446 & 0.212257 \\
\hline 863.693208 & 0.135617 & 0.029360 \\
\hline 869.660040 & 0.034724 & 0.007569 \\
\hline 877.994248 & 1.465719 & 0.322567 \\
\hline 904.656435 & 0.055122 & 0.012499 \\
\hline 915.512461 & 0.779600 & 0.178902 \\
\hline 918.581997 & 0.000000 & 0.000000 \\
\hline 926.619163 & 11.372079 & 2.641309 \\
\hline 936.554852 & 16.461056 & 3.864284 \\
\hline 969.786308 & 0.000000 & 0.000000 \\
\hline 1013.706853 & 15.246634 & 3.874043 \\
\hline 1019.573541 & 0.000000 & 0.000000 \\
\hline 1021.584887 & 7.625502 & 1.952634 \\
\hline 1025.063161 & 93.614490 & 24.053140 \\
\hline 1051.842904 & 0.013730 & 0.003620 \\
\hline 1056.095359 & 7.570806 & 2.004118 \\
\hline 1120.092931 & 1.673745 & 0.469917 \\
\hline 1145.112431 & 7.012307 & 2.012737 \\
\hline 1156.948856 & 3.486963 & 1.011206 \\
\hline 1180.447383 & 0.000000 & 0.000000 \\
\hline 1217.447524 & 0.786367 & 0.239968 \\
\hline 1225.876970 & 2.489837 & 0.765061 \\
\hline 1267.708628 & 108.031122 & 34.327834 \\
\hline 1334.576777 & 43.072822 & 14.408705 \\
\hline 1338.538721 & 0.000000 & 0.000000 \\
\hline 1352.787939 & 67.310390 & 22.823902 \\
\hline 1367.367267 & 18.207899 & 6.240553 \\
\hline 1397.922768 & 0.000000 & 0.000000 \\
\hline 1399.128539 & 75.972711 & 26.643625 \\
\hline 1405.292305 & 0.905350 & 0.318905 \\
\hline 1406.576021 & 0.082289 & 0.029013 \\
\hline
\end{tabular}




$\begin{array}{lcl}1407.570382 & 7.301485 & 2.576080 \\ 1409.232340 & 0.000000 & 0.000000 \\ 1417.999796 & 0.074293 & 0.026406 \\ 1429.297000 & 3.368553 & 1.206824 \\ 1434.816042 & 69.390554 & 24.955981 \\ 1443.994870 & 43.716462 & 15.822996 \\ 1922.380402 & 2.388385 & 1.150858 \\ 1972.397877 & 1.302835 & 0.644113 \\ 1988.114326 & 8.782048 & 4.376382 \\ 2951.330872 & 10.650040 & 7.878566 \\ 2952.714910 & 0.146164 & 0.108178 \\ 3035.048606 & 3.690563 & 2.807607 \\ 3035.486083 & 7.140766 & 5.433143 \\ 3037.726946 & 0.000000 & 0.000000 \\ 3038.118253 & 0.268855 & 0.204739 \\ 3151.445254 & 23.228389 & 18.348769 \\ 3151.668292 & 0.000000 & 0.000000 \\ 3154.091751 & 10.191612 & 8.057406 \\ 3154.861587 & 23.859631 & 18.867837 \\ 3158.907736 & 0.000000 & 0.000000 \\ 3161.397834 & 102.434454 & 81.171445 \\ 3170.006397 & 9.103734 & 7.233654 \\ 3170.065464 & 35.513285 & 28.218709\end{array}$

\section{1- $\mathrm{C}_{S}$ transition state}

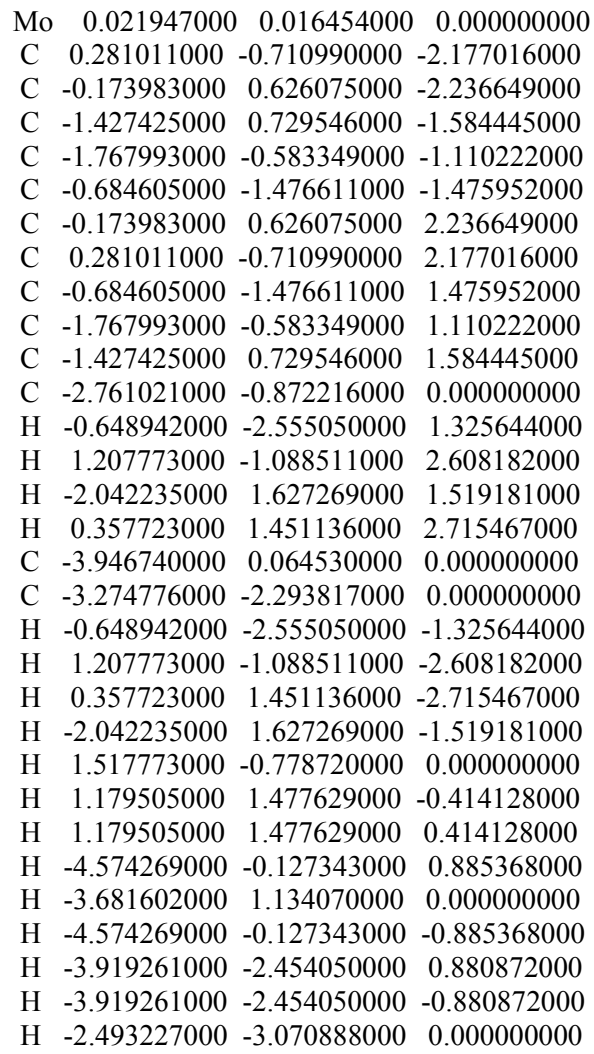

Frequencies

$\begin{array}{ccc}\begin{array}{c}\text { Frequency } \\ \text { cm-1 }\end{array} & \begin{array}{c}\text { Dipole Strength } \\ 1 \mathrm{e}-40 \text { esu2 cm2 }\end{array} & \begin{array}{c}\text { Absorption Intensity (degeneracy not counted) } \\ \mathrm{km} / \text { mole }\end{array} \\ ------ & ---- & - \\ -800.714985 & 2.837129 & -0.569423 \\ 109.948423 & 80.249685 & 2.211620 \\ 173.679160 & 0.370183 & 0.016115\end{array}$




\begin{tabular}{|c|c|c|}
\hline 182.350682 & 27.435939 & 1.254022 \\
\hline 214.537625 & 34.756534 & 1.869037 \\
\hline 220.649755 & 8.715949 & 0.482055 \\
\hline 244.987209 & 66.750132 & 4.098959 \\
\hline 264.856405 & 204.470963 & 13.574386 \\
\hline 267.248437 & 3.743415 & 0.250762 \\
\hline 302.403438 & 25.654991 & 1.944629 \\
\hline 309.951026 & 32.686600 & 2.539457 \\
\hline 357.703120 & 584.781315 & 52.431742 \\
\hline 366.462988 & 15.139146 & 1.390623 \\
\hline 417.965634 & 79.587510 & 8.338032 \\
\hline 429.063253 & 284.678684 & 30.616415 \\
\hline 438.035353 & 35.839248 & 3.935013 \\
\hline 451.627615 & 27.378501 & 3.099333 \\
\hline 492.585001 & 87.616294 & 10.817929 \\
\hline 508.995366 & 95.553470 & 12.190973 \\
\hline 549.005384 & 0.850535 & 0.117043 \\
\hline 596.047748 & 0.257884 & 0.038529 \\
\hline 607.810094 & 19.502951 & 2.971299 \\
\hline 608.497463 & 0.651945 & 0.099437 \\
\hline 700.215914 & 165.308840 & 29.013901 \\
\hline 719.311138 & 71.010731 & 12.803209 \\
\hline 728.237828 & 55.951493 & 10.213227 \\
\hline 740.612706 & 9.408667 & 1.746616 \\
\hline 782.670358 & 0.000001 & 0.000000 \\
\hline 804.377434 & 7.721041 & 1.556732 \\
\hline 805.005297 & 80.007918 & 16.143949 \\
\hline 816.125545 & 27.999419 & 5.727750 \\
\hline 833.522214 & 7.334207 & 1.532316 \\
\hline 840.346912 & 5.963921 & 1.256228 \\
\hline 852.247084 & 84.349912 & 18.018898 \\
\hline 859.869410 & 13.915548 & 2.999238 \\
\hline 874.862512 & 0.236961 & 0.051963 \\
\hline 884.851912 & 0.606213 & 0.134454 \\
\hline 911.999922 & 11.883812 & 2.716618 \\
\hline 916.870522 & 4.556624 & 1.047199 \\
\hline 925.296722 & 27.137402 & 6.294009 \\
\hline 927.426377 & 12.879433 & 2.994017 \\
\hline 963.821725 & 0.017850 & 0.004312 \\
\hline 1005.369419 & 11.638114 & 2.932826 \\
\hline 1013.145849 & 0.184746 & 0.046916 \\
\hline 1013.719123 & 25.951588 & 6.594162 \\
\hline 1020.052757 & 98.656725 & 25.224781 \\
\hline 1045.677216 & 2.601831 & 0.681954 \\
\hline 1048.903425 & 13.496070 & 3.548305 \\
\hline 1107.412466 & 151.298429 & 41.997351 \\
\hline 1109.915561 & 7.323942 & 2.037572 \\
\hline 1133.738572 & 0.430245 & 0.122266 \\
\hline 1142.052391 & 3.434920 & 0.983288 \\
\hline 1171.115848 & 0.034195 & 0.010038 \\
\hline 1215.764147 & 3.262870 & 0.994322 \\
\hline 1221.466336 & 0.104043 & 0.031855 \\
\hline 1275.312431 & 105.238222 & 33.640943 \\
\hline 1305.434314 & 1.002989 & 0.328193 \\
\hline 1330.202891 & 16.079931 & 5.361424 \\
\hline 1349.333853 & 39.956748 & 13.514115 \\
\hline 1354.456156 & 64.196683 & 21.794937 \\
\hline 1392.775368 & 3.246541 & 1.133392 \\
\hline 1394.161026 & 2.214551 & 0.773885 \\
\hline 1400.919296 & 33.951671 & 11.922088 \\
\hline 1402.226213 & 33.889279 & 11.911281 \\
\hline 1405.518635 & 19.005533 & 6.695681 \\
\hline 1406.092757 & 3.567911 & 1.257494 \\
\hline 1412.303868 & 10.976076 & 3.885558 \\
\hline 1414.738036 & 1.555057 & 0.551443 \\
\hline 1416.668729 & 92.726347 & 32.926800 \\
\hline 1434.148140 & 67.402932 & 24.229858 \\
\hline
\end{tabular}




$\begin{array}{cccl} & 1439.661551 & 30.125130 & 10.870948 \\ 1912.675541 & 42.515584 & 20.382973 \\ 2948.739090 & 7.318874 & 5.409520 \\ 2955.616732 & 4.713424 & 3.491907 \\ 3024.013785 & 0.000105 & 0.000079 \\ 3035.538251 & 5.199339 & 3.956051 \\ 3037.210486 & 0.029849 & 0.022724 \\ 3037.338021 & 5.825812 & 4.435347 \\ 3133.565896 & 6.691696 & 5.255973 \\ 3134.014821 & 19.492308 & 15.312369 \\ 3155.739956 & 3.927136 & 3.106384 \\ 3156.076849 & 40.445618 & 31.996105 \\ 3156.757683 & 2.799940 & 2.215481 \\ 3156.841473 & 51.217135 & 40.527156 \\ & 3.739033 & 2.968679 \\ & 3167.567367 & 2.069292 \\ 3167.765137 & 34.091480 & 27.06908 \\ & 3338.266378 & 2.274985 & 1.903608 \\ & & & \end{array}$

\section{Coordinates}

$\begin{array}{llll}\text { Mo } & -0.035011000 & 0.100607000 & -0.048738000\end{array}$

$\begin{array}{llll}\text { C } & 2.264075000 & -0.185272000 & 0.199653000\end{array}$

$\begin{array}{llll}\text { C } & 1.962925000 & 1.199195000 & 0.240847000\end{array}$

C $\quad 1.403380000 \quad 1.569020000-1.017790000$

$\begin{array}{lllll}\text { C } & 1.308933000 & 0.405306000 & -1.837654000\end{array}$

C $\quad 1.857273000 \quad-0.671742000-1.060860000$

$\begin{array}{llll}\text { C } & -2.290530000 & -0.243294000 & 0.232116000\end{array}$

$\begin{array}{llll}\text { C } & -1.687617000 & -1.525597000 & 0.191931000\end{array}$

C $-1.063908000-1.663144000-1.066163000$

C $-1.280237000-0.474014000-1.842451000$

$\begin{array}{llll}\text { C } & -2.065743000 & 0.391902000 & -1.024844000\end{array}$

Si $\quad-0.027753000 \quad 0.095891000 \quad-3.158814000$

H $\quad-0.495086000 \quad-2.536071000-1.391210000$

$\mathrm{H} \quad-1.719747000 \quad-2.273978000 \quad 0.983964000$

H $\quad-2.440636000 \quad 1.375069000 \quad-1.312730000$

$\begin{array}{llll}\mathrm{H} & -2.862420000 & 0.165683000 & 1.064414000\end{array}$

C $\quad-0.567018000 \quad 1.680345000 \quad-3.936947000$

$\begin{array}{lllll}\text { C } & 0.436044000 & -1.303999000 & -4.267333000\end{array}$

H $\quad 1.938050000 \quad-1.710361000-1.386811000$

$\begin{array}{llll}\mathrm{H} & 2.741323000 & -0.761404000 & 0.992596000\end{array}$

$\begin{array}{llll}\mathrm{H} & 2.163985000 & 1.870673000 & 1.074908000\end{array}$

$\mathrm{H} \quad 1.104015000 \quad 2.577977000 \quad-1.304690000$

$\begin{array}{llll}\mathrm{H} & 0.246302000 & -0.734514000 & 1.384166000\end{array}$

$\begin{array}{llll}\mathrm{H} & -0.568213000 & 1.673028000 & 0.115935000\end{array}$

$\begin{array}{lllll}\mathrm{H} & -0.287052000 & 0.835988000 & 1.457893000\end{array}$

$\begin{array}{llll}\mathrm{H} & 1.274183000 & -1.031801000 & -4.932543000\end{array}$

H $\quad 0.735958000 \quad-2.207503000-3.708554000$

H $\quad-0.422976000 \quad-1.582380000-4.904806000$

$\begin{array}{lllll}\mathrm{H} & -0.832552000 & 2.447165000 & -3.189305000\end{array}$

$\mathrm{H} \quad 0.230698000 \quad 2.098542000 \quad-4.575263000$

H $\quad-1.450411000 \quad 1.521487000 \quad-4.579771000$

\section{Frequencies}

$\begin{array}{ccc}\begin{array}{c}\text { Frequency } \\ \mathrm{cm}-1\end{array} & \begin{array}{c}\text { Dipole Strength } \\ 1 \mathrm{e}-40 \mathrm{esu} 2 \mathrm{~cm} 2\end{array} & \begin{array}{c}\text { Absorption Intensity (degeneracy not counted) } \\ \mathrm{km} / \mathrm{mole}\end{array} \\ ------ & --- & 0.711105 \\ 76.430550 & 37.118368 & 0.005430 \\ 99.023745 & 0.218762 & 0.815185 \\ 130.078316 & 25.001913 & 0.019965 \\ 136.225670 & 0.584703 & 0.053759 \\ 148.497940 & 1.444290 & 1.060036 \\ 151.089778 & 27.990295 & 0.005282 \\ 175.334344 & 0.120177 & 7.353597 \\ 211.892518 & 138.454195 & 7.637016 \\ 212.565274 & 143.335357 & 10.846614 \\ 297.568402 & 145.421729 & \end{array}$




\begin{tabular}{|c|c|c|}
\hline 312.347977 & 144.206682 & 11.290213 \\
\hline 337.455374 & 138.432768 & 11.709364 \\
\hline 366.752932 & 8.598484 & 0.790448 \\
\hline 388.093311 & 61.277755 & 5.960972 \\
\hline 399.305713 & 41.099728 & 4.113605 \\
\hline 404.835464 & 72.226185 & 7.329111 \\
\hline 421.046988 & 26.249420 & 2.770310 \\
\hline 456.327170 & 122.606352 & 14.023854 \\
\hline 473.583175 & 9.291779 & 1.102994 \\
\hline 596.377371 & 16.766820 & 2.506397 \\
\hline 607.806499 & 12.523542 & 1.907966 \\
\hline 645.199962 & 232.089341 & 37.534238 \\
\hline 649.790802 & 186.812863 & 30.426949 \\
\hline 668.409919 & 108.537888 & 18.184540 \\
\hline 671.818867 & 0.995060 & 0.167564 \\
\hline 692.101139 & 137.309866 & 23.820418 \\
\hline 699.189946 & 102.956584 & 18.043774 \\
\hline 720.149136 & 1.665347 & 0.300611 \\
\hline 728.070722 & 289.945046 & 52.913603 \\
\hline 768.497519 & 126.846163 & 24.434186 \\
\hline 786.061875 & 106.406406 & 20.965372 \\
\hline 802.432466 & 257.323738 & 51.756680 \\
\hline 812.849273 & 291.646761 & 59.421724 \\
\hline 813.245874 & 420.505616 & 85.717943 \\
\hline 834.776606 & 55.368353 & 11.585371 \\
\hline 839.964899 & 101.672635 & 21.406383 \\
\hline 845.627776 & 1.807626 & 0.383147 \\
\hline 849.212939 & 19.362200 & 4.121444 \\
\hline 850.831254 & 3.341357 & 0.712598 \\
\hline 854.493546 & 9.949299 & 2.130980 \\
\hline 877.199584 & 15.136329 & 3.328105 \\
\hline 880.848062 & 77.087927 & 17.020231 \\
\hline 903.526128 & 62.204775 & 14.087778 \\
\hline 916.646136 & 13.796982 & 3.170034 \\
\hline 919.395246 & 1.191784 & 0.274649 \\
\hline 930.190596 & 3.494324 & 0.814729 \\
\hline 1008.851036 & 26.510486 & 6.703827 \\
\hline 1012.373976 & 128.950394 & 32.722141 \\
\hline 1021.436805 & 4.053553 & 1.037828 \\
\hline 1029.576181 & 91.929607 & 23.724221 \\
\hline 1045.340041 & 0.095026 & 0.024899 \\
\hline 1050.803259 & 15.994933 & 4.212907 \\
\hline 1155.455195 & 5.463779 & 1.582429 \\
\hline 1156.501532 & 127.139991 & 36.855852 \\
\hline 1170.749999 & 1.990096 & 0.584005 \\
\hline 1175.339878 & 17.962022 & 5.291719 \\
\hline 1226.328036 & 222.068208 & 68.260759 \\
\hline 1229.839250 & 108.743403 & 33.521955 \\
\hline 1308.582325 & 3.148826 & 1.032828 \\
\hline 1320.554769 & 80.542860 & 26.660085 \\
\hline 1361.317122 & 0.825614 & 0.281718 \\
\hline 1365.846142 & 9.249956 & 3.166792 \\
\hline 1371.133510 & 33.262101 & 11.431612 \\
\hline 1374.720423 & 0.964278 & 0.332273 \\
\hline 1375.141478 & 11.207197 & 3.862980 \\
\hline 1375.588952 & 24.189297 & 8.340460 \\
\hline 1399.994277 & 1.531791 & 0.537531 \\
\hline 1400.560254 & 34.783713 & 12.211129 \\
\hline 1413.293670 & 12.427446 & 4.402429 \\
\hline 1414.801478 & 2.950533 & 1.046343 \\
\hline 1957.109484 & 4.732809 & 2.321733 \\
\hline 1981.325505 & 0.349947 & 0.173795 \\
\hline 2016.569018 & 2.315954 & 1.170634 \\
\hline 2937.704178 & 6.112260 & 4.500781 \\
\hline 2943.985251 & 7.007004 & 5.170661 \\
\hline 3024.015573 & 2.101295 & 1.592755 \\
\hline 3030.923984 & 2.702416 & 2.053077 \\
\hline
\end{tabular}




$\begin{array}{ccl}3032.388980 & 3.424455 & 2.602882 \\ 3033.614971 & 0.426182 & 0.324066 \\ 3135.420120 & 1.177050 & 0.925058 \\ 3138.091835 & 16.415593 & 12.912201 \\ 3145.450769 & 2.842747 & 2.241296 \\ 3146.756852 & 13.793802 & 10.879908 \\ 3156.101280 & 0.228352 & 0.180648 \\ 3157.092131 & 54.916395 & 43.457761 \\ 3167.695355 & 40.702099 & 32.317540 \\ 3167.749801 & 15.590673 & 12.379235\end{array}$

\section{2- $\mathrm{C}_{2 \mathrm{~V}}$}

\section{Coordinates}

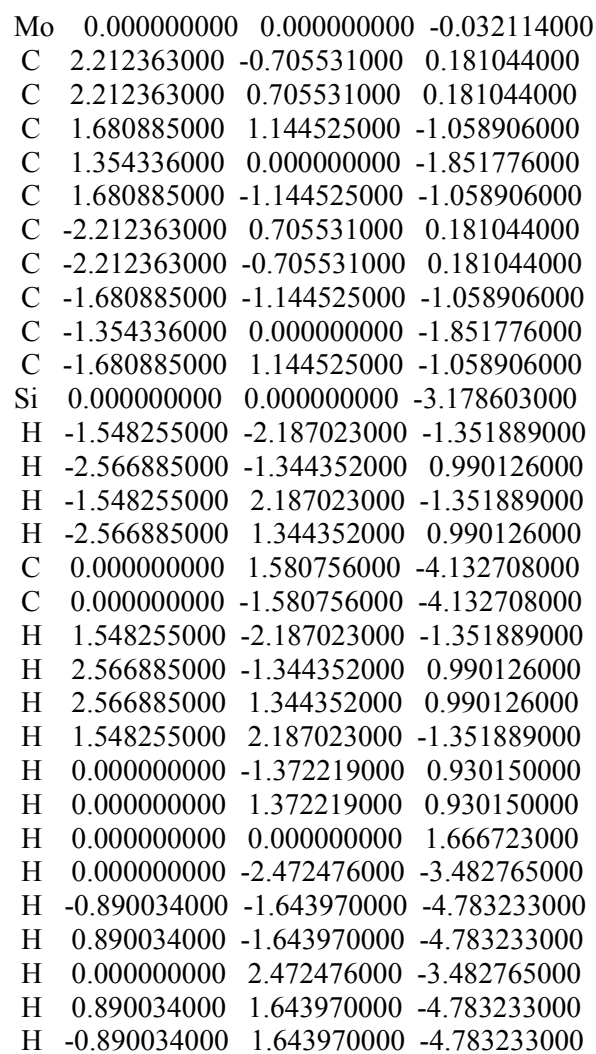

\section{Frequencies}

$\begin{array}{ccc}\begin{array}{c}\text { Frequency } \\ \text { cm-1 }\end{array} & \begin{array}{c}\text { Dipole Strength } \\ 1 \mathrm{e}-40 \text { esu2 } \mathrm{cm} 2\end{array} & \begin{array}{c}\text { Absorption Intensity (degeneracy not counted) } \\ \mathrm{km} / \mathrm{mole}\end{array} \\ -119.389581 & 0.412690 & -0.012350 \\ 74.859608 & 14.626608 & 0.274454 \\ 92.450497 & 0.000000 & 0.000000 \\ 137.520616 & 0.000000 & 0.000000 \\ 138.539335 & 3.487697 & 0.121113 \\ 151.818735 & 36.102964 & 1.373872 \\ 175.715896 & 0.685869 & 0.030209 \\ 201.013442 & 0.000000 & 0.000000 \\ 217.895892 & 369.814300 & 20.198114 \\ 252.978638 & 21.915740 & 1.389690 \\ 299.133101 & 355.125161 & 26.627107 \\ 312.108175 & 9.819139 & 0.768169 \\ 372.439659 & 0.000000 & 0.000000 \\ 373.567066 & 22.316361 & 2.089633\end{array}$




\begin{tabular}{|c|c|c|}
\hline 397.018563 & 338.741385 & 33.709875 \\
\hline 398.652062 & 126.401212 & 12.630581 \\
\hline 408.889752 & 91.029048 & 9.329627 \\
\hline 456.894939 & 86.710570 & 9.930393 \\
\hline 463.986339 & 68.486143 & 7.965003 \\
\hline 559.126107 & 153.224668 & 21.474177 \\
\hline 599.371731 & 0.000000 & 0.000000 \\
\hline 612.764257 & 0.542938 & 0.083392 \\
\hline 619.010498 & 77.328333 & 11.998162 \\
\hline 622.478750 & 515.470714 & 80.427875 \\
\hline 668.001192 & 92.761216 & 15.531798 \\
\hline 671.489986 & 5.250860 & 0.883788 \\
\hline 704.393733 & 237.846493 & 41.994296 \\
\hline 712.448318 & 300.010113 & 53.575635 \\
\hline 718.191612 & 0.000000 & 0.000000 \\
\hline 779.463401 & 1.703741 & 0.332872 \\
\hline 780.568863 & 0.000000 & 0.000000 \\
\hline 801.143599 & 277.742530 & 55.773876 \\
\hline 801.347082 & 623.657319 & 125.269330 \\
\hline 814.405244 & 380.345517 & 77.642039 \\
\hline 831.903863 & 15.000036 & 3.127833 \\
\hline 833.561197 & 87.190089 & 18.217244 \\
\hline 837.011755 & 0.000000 & 0.000000 \\
\hline 855.958364 & 3.116818 & 0.668717 \\
\hline 857.607107 & 0.000000 & 0.000000 \\
\hline 867.533287 & 7.253304 & 1.577249 \\
\hline 871.292905 & 32.132076 & 7.017478 \\
\hline 874.266466 & 22.078133 & 4.838205 \\
\hline 897.727408 & 8.473419 & 1.906695 \\
\hline 901.950020 & 129.309997 & 29.234296 \\
\hline 919.261044 & 0.024053 & 0.005542 \\
\hline 929.065649 & 0.000000 & 0.000000 \\
\hline 1015.623704 & 0.000000 & 0.000000 \\
\hline 1018.946653 & 17.809457 & 4.548626 \\
\hline 1021.393566 & 53.180363 & 13.615152 \\
\hline 1022.063067 & 140.599092 & 36.019550 \\
\hline 1051.677489 & 0.105777 & 0.027884 \\
\hline 1054.814530 & 38.126943 & 10.080595 \\
\hline 1163.865131 & 4.929714 & 1.438144 \\
\hline 1165.160613 & 134.054406 & 39.151189 \\
\hline 1168.733832 & 0.000000 & 0.000000 \\
\hline 1172.922915 & 6.911630 & 2.032020 \\
\hline 1226.225553 & 221.083684 & 67.952451 \\
\hline 1229.281594 & 99.516497 & 30.663698 \\
\hline 1312.683102 & 0.000000 & 0.000000 \\
\hline 1324.958081 & 64.287127 & 21.350312 \\
\hline 1360.937004 & 0.000000 & 0.000000 \\
\hline 1366.254277 & 10.977543 & 3.759367 \\
\hline 1373.353635 & 19.112541 & 6.579288 \\
\hline 1375.075151 & 28.699804 & 9.891985 \\
\hline 1386.483605 & 0.088776 & 0.030852 \\
\hline 1389.718522 & 0.825614 & 0.287595 \\
\hline 1393.732983 & 52.406214 & 18.307980 \\
\hline 1394.254306 & 0.000000 & 0.000000 \\
\hline 1421.301010 & 4.499026 & 1.602812 \\
\hline 1421.548644 & 9.590854 & 3.417410 \\
\hline 1947.187710 & 0.003929 & 0.001918 \\
\hline 1994.711365 & 2.583067 & 1.291498 \\
\hline 1998.896292 & 4.490658 & 2.249978 \\
\hline 2941.891359 & 1.060688 & 0.782154 \\
\hline 2942.874084 & 12.299328 & 9.072587 \\
\hline 3030.809599 & 7.930661 & 6.024848 \\
\hline 3030.995337 & 0.000000 & 0.000000 \\
\hline 3031.800994 & 0.345109 & 0.262262 \\
\hline 3032.488785 & 0.638569 & 0.485384 \\
\hline 3142.180797 & 0.000000 & 0.000000 \\
\hline 3142.532654 & 18.540302 & 14.604096 \\
\hline
\end{tabular}




$\begin{array}{ccl}3144.546931 & 2.914061 & 2.296861 \\ 3145.115218 & 15.018832 & 11.839975 \\ 3153.311260 & 0.000000 & 0.000000 \\ 3154.662978 & 61.658746 & 48.755737 \\ 3165.042514 & 40.873082 & 32.426121 \\ 3165.080387 & 5.086417 & 4.035290\end{array}$

\section{2-C $\mathrm{C}_{\text {S }}$ transition state}

\section{Coordinates}

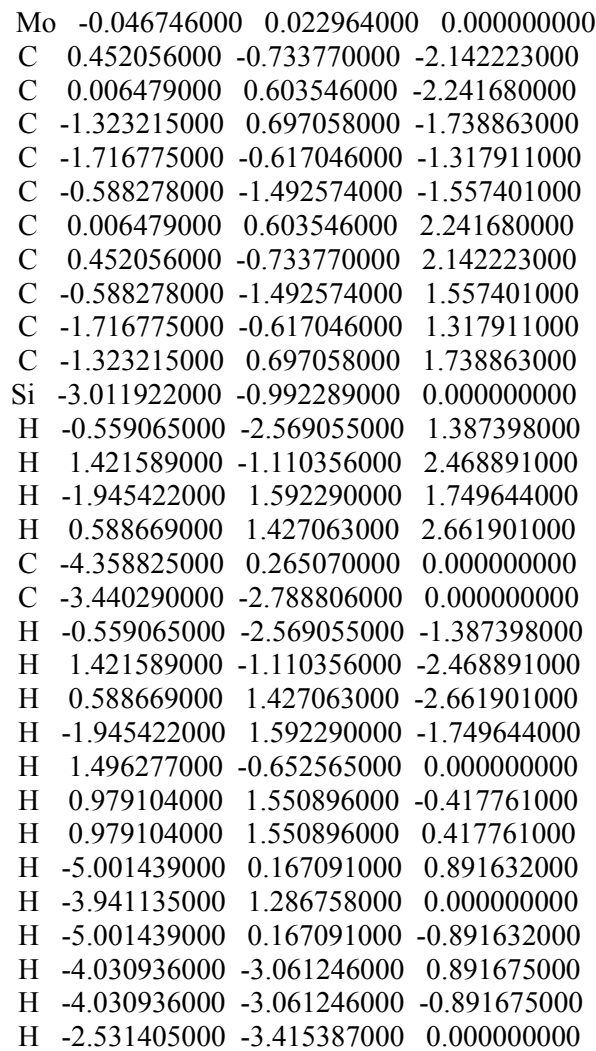

\section{Frequencies}

\begin{tabular}{|c|c|c|}
\hline $\begin{array}{l}\text { Frequency } \\
\text { cm-1 }\end{array}$ & $\begin{array}{l}\text { Dipole Strength } \\
1 \mathrm{e}-40 \text { esu } 2 \mathrm{~cm} 2\end{array}$ & $\begin{array}{l}\text { Absorption Intensity (degeneracy not counted) } \\
\mathrm{km} / \mathrm{mole}\end{array}$ \\
\hline-828.073963 & 1.283948 & -0.266498 \\
\hline 78.348890 & 16.647694 & 0.326938 \\
\hline 93.314652 & 9.322619 & 0.218055 \\
\hline 116.968157 & 66.645160 & 1.953954 \\
\hline 137.526075 & 17.441372 & 0.601234 \\
\hline 143.340835 & 0.402308 & 0.014455 \\
\hline 152.084824 & 22.279496 & 0.849316 \\
\hline 163.809354 & 14.201061 & 0.583093 \\
\hline 209.709131 & 28.626543 & 1.504750 \\
\hline 213.224881 & 159.371515 & 8.517785 \\
\hline 247.484355 & 312.928374 & 19.412021 \\
\hline 304.622499 & 237.560020 & 18.139003 \\
\hline 320.889048 & 5.083282 & 0.408862 \\
\hline 365.944916 & 258.132624 & 23.677546 \\
\hline 394.705732 & 15.202872 & 1.504101 \\
\hline 397.925579 & 337.859242 & 33.698900 \\
\hline 415.152918 & 70.653783 & 7.352272 \\
\hline 425.218929 & 261.249573 & 27.844938 \\
\hline
\end{tabular}




\begin{tabular}{|c|c|c|}
\hline 444.333856 & 15.567883 & 1.733872 \\
\hline 466.603226 & 12.572076 & 1.470391 \\
\hline 485.999833 & 227.456778 & 27.708504 \\
\hline 592.398777 & 4.868907 & 0.722976 \\
\hline 605.244577 & 12.486782 & 1.894347 \\
\hline 651.284099 & 7.337498 & 1.197834 \\
\hline 663.430589 & 110.632364 & 18.397370 \\
\hline 674.758835 & 6.021850 & 1.018489 \\
\hline 680.577853 & 12.966986 & 2.212050 \\
\hline 716.488156 & 0.387861 & 0.069657 \\
\hline 724.395319 & 17.760058 & 3.224765 \\
\hline 746.454151 & 318.172592 & 59.531109 \\
\hline 773.122763 & 56.275790 & 10.905564 \\
\hline 790.879992 & 29.691154 & 5.885939 \\
\hline 804.334089 & 267.514182 & 53.933842 \\
\hline 807.914091 & 80.725818 & 16.347664 \\
\hline 812.367070 & 247.463404 & 50.389652 \\
\hline 822.617625 & 6.482539 & 1.336661 \\
\hline 826.683392 & 213.456106 & 44.230909 \\
\hline 836.784538 & 0.004231 & 0.000888 \\
\hline 845.574733 & 7.459719 & 1.581075 \\
\hline 864.304363 & 13.955107 & 3.023277 \\
\hline 868.870716 & 130.232861 & 28.363107 \\
\hline 885.276186 & 0.271580 & 0.060264 \\
\hline 896.958960 & 70.878515 & 15.935484 \\
\hline 916.867449 & 7.374983 & 1.694906 \\
\hline 927.127382 & 8.140272 & 1.891718 \\
\hline 1002.525904 & 6.618007 & 1.663033 \\
\hline 1009.173406 & 134.606092 & 34.049331 \\
\hline 1013.727051 & 3.190686 & 0.810743 \\
\hline 1022.109489 & 116.672518 & 29.891249 \\
\hline 1043.752661 & 4.095933 & 1.071590 \\
\hline 1047.125142 & 49.207005 & 12.915273 \\
\hline 1147.474586 & 50.119800 & 14.415525 \\
\hline 1149.642099 & 0.004772 & 0.001375 \\
\hline 1160.329999 & 206.892619 & 60.173405 \\
\hline 1160.545515 & 0.247285 & 0.071935 \\
\hline 1165.668916 & 22.682919 & 6.627538 \\
\hline 1223.485295 & 234.304556 & 71.855093 \\
\hline 1226.570197 & 102.176852 & 31.413982 \\
\hline 1285.418791 & 1.940422 & 0.625200 \\
\hline 1303.496953 & 58.217553 & 19.021381 \\
\hline 1362.251231 & 0.040402 & 0.013795 \\
\hline 1366.049435 & 9.261529 & 3.171226 \\
\hline 1366.280966 & 6.314191 & 2.162399 \\
\hline 1372.967286 & 2.378928 & 0.818690 \\
\hline 1374.558398 & 14.754138 & 5.083410 \\
\hline 1375.956283 & 24.002325 & 8.278202 \\
\hline 1392.932881 & 1.656244 & 0.578272 \\
\hline 1395.403683 & 38.655397 & 13.520354 \\
\hline 1409.928902 & 12.652740 & 4.471568 \\
\hline 1412.646481 & 4.646876 & 1.645405 \\
\hline 1474.967968 & 94.904625 & 35.087146 \\
\hline 1933.817685 & 18.892001 & 9.157387 \\
\hline 2942.770161 & 3.409443 & 2.514883 \\
\hline 2944.975599 & 7.707493 & 5.689485 \\
\hline 3029.720304 & 0.549657 & 0.417419 \\
\hline 3032.531798 & 0.604114 & 0.459201 \\
\hline 3036.086002 & 3.881211 & 2.953653 \\
\hline 3038.735415 & 3.511810 & 2.674866 \\
\hline 3126.670872 & 4.168451 & 3.266893 \\
\hline 3127.506911 & 17.645021 & 13.832432 \\
\hline 3146.130611 & 2.526783 & 1.992612 \\
\hline 3146.929797 & 17.743719 & 13.996188 \\
\hline 3151.096007 & 0.015077 & 0.011908 \\
\hline 3152.397475 & 39.152680 & 30.937167 \\
\hline 3161.847324 & 2.668503 & 2.114884 \\
\hline
\end{tabular}




$\begin{array}{lcl}3162.169560 & 28.031527 & 22.218257 \\ 3250.527991 & 1.389187 & 1.131860\end{array}$

3

\section{Coordinates}

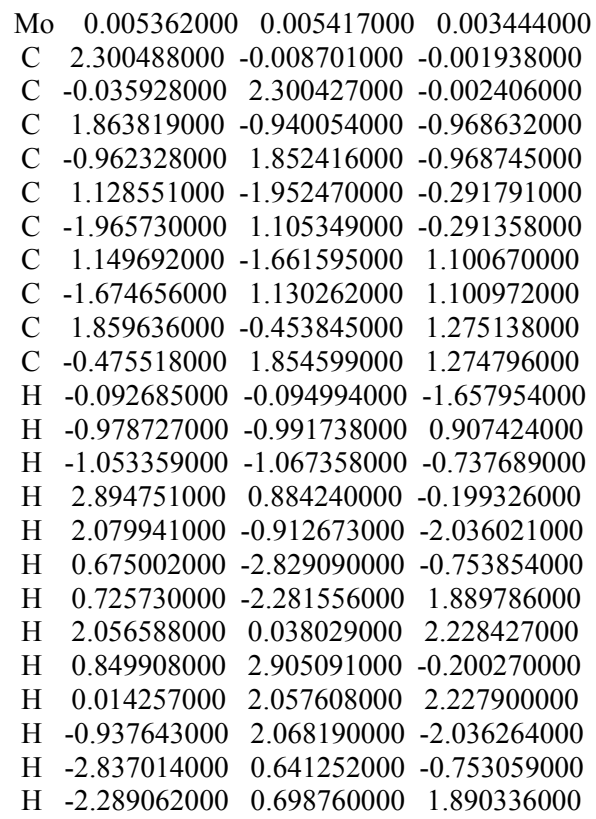

Frequencies

\begin{tabular}{|c|c|c|}
\hline $\begin{array}{l}\text { Frequency } \\
\mathrm{cm}-1\end{array}$ & $\begin{array}{l}\text { Dipole Strength } \\
1 \mathrm{e}-40 \text { esu } 2 \mathrm{~cm} 2\end{array}$ & $\begin{array}{l}\text { Absorption Intensity (degeneracy not counted) } \\
\mathrm{km} / \mathrm{mole}\end{array}$ \\
\hline 61.348252 & 1.185396 & 0.018228 \\
\hline 132.401637 & 94.832186 & 3.147221 \\
\hline 140.732762 & 107.677642 & 3.798384 \\
\hline 336.734993 & 26.724785 & 2.255696 \\
\hline 349.871667 & 36.511918 & 3.202001 \\
\hline 366.215724 & 74.946450 & 6.879646 \\
\hline 380.605323 & 1.249081 & 0.119164 \\
\hline 392.602735 & 142.098342 & 13.983645 \\
\hline 413.571806 & 176.524135 & 18.299241 \\
\hline 450.685129 & 9.160691 & 1.034855 \\
\hline 599.622309 & 19.396953 & 2.915341 \\
\hline 609.895994 & 2.345219 & 0.358523 \\
\hline 612.320657 & 1.954071 & 0.299914 \\
\hline 619.452930 & 2.275652 & 0.353340 \\
\hline 718.137713 & 730.232615 & 131.445896 \\
\hline 747.883205 & 118.315433 & 22.179576 \\
\hline 781.923620 & 212.640592 & 41.676244 \\
\hline 786.693307 & 309.934032 & 61.115700 \\
\hline 788.842077 & 40.105002 & 7.929881 \\
\hline 826.972322 & 18.268724 & 3.786843 \\
\hline 829.048316 & 25.308771 & 5.259312 \\
\hline 831.657503 & 1.656325 & 0.345277 \\
\hline 840.503999 & 5.735231 & 1.208283 \\
\hline 848.166334 & 12.584870 & 2.675518 \\
\hline 850.790049 & 42.356151 & 9.032687 \\
\hline 854.409339 & 18.074245 & 3.870831 \\
\hline 857.415437 & 1.732527 & 0.372348 \\
\hline 862.436015 & 32.511940 & 7.028260 \\
\hline 884.571471 & 16.558048 & 3.671302 \\
\hline
\end{tabular}




$\begin{array}{ccc}919.202210 & 0.845511 & 0.194809 \\ 926.812092 & 6.763170 & 1.571159 \\ 942.685001 & 0.352553 & 0.083305 \\ 949.078578 & 19.749149 & 4.698169 \\ 990.549942 & 5.465490 & 1.357012 \\ 991.027608 & 80.851370 & 20.084046 \\ 1002.860153 & 3.275952 & 0.823486 \\ 1004.699127 & 63.310298 & 15.943676 \\ 1037.690246 & 0.167049 & 0.043450 \\ 1044.066979 & 0.372350 & 0.097445 \\ 1049.206403 & 1.059235 & 0.278568 \\ 1049.968376 & 3.329737 & 0.876323 \\ 1130.779444 & 3.351324 & 0.949888 \\ 1131.745662 & 3.323380 & 0.942772 \\ 1226.810372 & 0.200118 & 0.061538 \\ 1228.279209 & 0.091333 & 0.028119 \\ 1382.817926 & 10.500822 & 3.639706 \\ 1382.825475 & 0.428795 & 0.148626 \\ 1383.980812 & 1.539663 & 0.534114 \\ 1396.843764 & 2.464745 & 0.862974 \\ 1412.868431 & 56.588685 & 20.040539 \\ 1413.834021 & 10.153644 & 3.598309 \\ 1428.403843 & 68.817624 & 24.639322 \\ 1431.071514 & 6.052755 & 2.171164 \\ 1993.394306 & 0.004179 & 0.002088 \\ 2014.294769 & 0.809353 & 0.408638 \\ 2015.157502 & 0.010542 & 0.005325 \\ 3144.541970 & 0.599460 & 0.472493 \\ 3146.696033 & 9.092169 & 7.171341 \\ 3153.083808 & 0.434721 & 0.343577 \\ 3154.067428 & 11.237528 & 8.884231 \\ 3156.664314 & 1.691958 & 1.338740 \\ 3157.486644 & 89.817240 & 71.085218 \\ 3165.052883 & 0.120801 & 0.095836 \\ 3166.799316 & 94.560006 & 75.059575 \\ 3173.362230 & 4.225520 & 3.361073 \\ 3174.235457 & 10.128393 & 8.058566\end{array}$

\section{3-Cs transition state}

\section{Coordinates}
$\begin{array}{lllll}\text { Mo } & -0.073456000 & 0.037648000 & -0.000011000\end{array}$
C $\quad 1.190985000 \quad 1.132392000 \quad 1.466660000$
$\begin{array}{llll}\text { C } & 1.191317000 & 1.131845000 & -1.466581000\end{array}$
$\begin{array}{llll}\text { C } & 1.224717000 & -0.241920000 & 1.830438000\end{array}$
$\begin{array}{llll}\text { C } & 1.224440000 & -0.242478000 & -1.830384000\end{array}$
$\begin{array}{lrrr}\text { C } & -0.108829000 & -0.585968000 & 2.213266000\end{array}$
$\begin{array}{llll}\text { C } & -0.109275000 & -0.585864000 & -2.213347000\end{array}$
$\begin{array}{llll}\text { C } & -0.940717000 & 0.550849000 & 2.098457000\end{array}$
$\begin{array}{lllll}\text { C } & -0.940660000 & 0.551321000 & -2.098455000\end{array}$
$\begin{array}{llll}\text { C } & -0.149803000 & 1.618135000 & 1.622386000\end{array}$
$\begin{array}{llll}\text { C } & -0.149293000 & 1.618210000 & -1.622253000\end{array}$
H $-1.748789000 \quad 0.090864000 \quad-0.000093000$
$\mathrm{H} \quad-0.382464000 \quad-1.759234000 \quad 0.419761000$
$\mathrm{H} \quad-0.382595000 \quad-1.759146000-0.420164000$
$\begin{array}{lllll}\mathrm{H} & 2.052616000 & 1.733982000 & 1.182306000\end{array}$
H $\quad 2.102767000 \quad-0.882858000 \quad 1.902408000$
$\begin{array}{llll}\mathrm{H} & -0.432519000 & -1.562778000 & 2.579492000\end{array}$
$\begin{array}{llll}\mathrm{H} & -2.001534000 & 0.594340000 & 2.345330000\end{array}$
$\begin{array}{llll}\mathrm{H} & -0.494437000 & 2.639577000 & 1.463011000\end{array}$
$\begin{array}{llll}\mathrm{H} & 2.053278000 & 1.732979000 & -1.182282000\end{array}$
$\mathrm{H} \quad-0.493535000 \quad 2.639809000-1.463031000$
$\begin{array}{llll}\mathrm{H} & 2.102248000 & -0.883700000 & -1.902727000\end{array}$
$\mathrm{H} \quad-0.433420000 \quad-1.562466000-2.579760000$
$\begin{array}{lllll}\mathrm{H} & -2.001465000 & 0.595207000 & -2.345372000\end{array}$ 


\section{Frequencies}

\begin{tabular}{|c|c|c|}
\hline $\begin{array}{l}\text { Frequency } \\
\mathrm{cm}-1\end{array}$ & $\begin{array}{l}\text { Dipole Strength } \\
1 \mathrm{e}-40 \text { esu } 2 \mathrm{~cm} 2\end{array}$ & $\begin{array}{l}\text { Absorption Intensity (degeneracy not counted) } \\
\mathrm{km} / \mathrm{mole}\end{array}$ \\
\hline 854227030 & ----- & -0235233 \\
\hline $\begin{array}{l}-854.227950 \\
55.186528\end{array}$ & $\begin{array}{l}1.098618 \\
3.662949\end{array}$ & $\begin{array}{l}-0.235233 \\
0.050669\end{array}$ \\
\hline 94.334066 & 184.052940 & 4.352005 \\
\hline 161.944298 & 42.453846 & 1.723300 \\
\hline 261.047762 & 234.530787 & 15.346096 \\
\hline 329.452264 & 16.548922 & 1.366596 \\
\hline 336.995796 & 19.408514 & 1.639437 \\
\hline 381.618155 & 40.367738 & 3.861371 \\
\hline 419.586661 & 104.158640 & 10.954563 \\
\hline 441.038830 & 165.811345 & 18.330282 \\
\hline 468.086489 & 345.898311 & 40.583791 \\
\hline 470.771357 & 720.986718 & 85.077627 \\
\hline 591.622879 & 11.432716 & 1.695401 \\
\hline 596.516832 & 24.999610 & 3.737954 \\
\hline 601.638148 & 37.780746 & 5.697495 \\
\hline 609.371584 & 7.124921 & 1.088279 \\
\hline 710.302291 & 24.413078 & 4.346541 \\
\hline 749.538642 & 343.160118 & 64.471668 \\
\hline 779.575878 & 114.399334 & 22.354243 \\
\hline 795.498584 & 79.818559 & 15.915539 \\
\hline 814.540836 & 3.011950 & 0.614948 \\
\hline 820.839492 & 10.329963 & 2.125373 \\
\hline 827.909530 & 24.394786 & 5.062417 \\
\hline 833.836258 & 6.221447 & 1.300320 \\
\hline 835.880042 & 14.148583 & 2.964387 \\
\hline 837.319664 & 16.734525 & 3.512229 \\
\hline 862.994868 & 11.337435 & 2.452455 \\
\hline 865.961218 & 17.717255 & 3.845678 \\
\hline 902.057436 & 18.160304 & 4.106155 \\
\hline 904.746500 & 28.019188 & 6.354195 \\
\hline 923.622974 & 4.756271 & 1.101132 \\
\hline 941.473034 & 9.083108 & 2.143485 \\
\hline 974.921511 & 3.585657 & 0.876227 \\
\hline 979.283284 & 97.542627 & 23.943126 \\
\hline 992.838226 & 2.279473 & 0.567271 \\
\hline 1000.133135 & 77.526342 & 19.435028 \\
\hline 1030.370357 & 0.226159 & 0.058410 \\
\hline 1039.208015 & 5.466431 & 1.423916 \\
\hline 1040.842551 & 2.739325 & 0.714672 \\
\hline 1046.562359 & 8.558192 & 2.245046 \\
\hline 1119.952270 & 1.379799 & 0.387341 \\
\hline 1121.929102 & 8.738806 & 2.457512 \\
\hline 1193.455870 & 96.958577 & 29.004853 \\
\hline 1219.271455 & 0.253794 & 0.077564 \\
\hline 1219.867658 & 0.417350 & 0.127612 \\
\hline 1355.004815 & 6.544795 & 2.222875 \\
\hline 1369.526170 & 14.518899 & 4.984047 \\
\hline 1373.213087 & 2.353899 & 0.810222 \\
\hline 1377.377204 & 26.341435 & 9.094323 \\
\hline 1410.327533 & 20.064124 & 7.092809 \\
\hline 1413.650292 & 26.746196 & 9.477244 \\
\hline 1420.034604 & 49.900853 & 17.761718 \\
\hline 1421.494986 & 11.176198 & 3.982149 \\
\hline 1512.020440 & 102.843512 & 38.977379 \\
\hline 1954.860205 & 9.165274 & 4.490960 \\
\hline 3130.188843 & 5.976602 & 4.689246 \\
\hline 3130.938993 & 26.665160 & 20.926513 \\
\hline 3152.458798 & 0.313761 & 0.247928 \\
\hline 3154.095159 & 15.726818 & 12.433509 \\
\hline
\end{tabular}




$\begin{array}{lcl}3158.477369 & 3.754103 & 2.972090 \\ 3162.028025 & 39.579605 & 31.370052 \\ 3162.912741 & 2.623678 & 2.080060 \\ 3164.719519 & 95.778606 & 75.976942 \\ 3172.718264 & 3.481685 & 2.768847 \\ 3174.553947 & 8.782230 & 6.988205 \\ 3199.238163 & 0.606291 & 0.486189\end{array}$

\section{Thermodynamic information}

\begin{tabular}{l|r|r|r|r|r} 
Species & \multicolumn{1}{|c|}{$\mathbf{E}_{\mathbf{S C F}}$} & $\mathbf{E}_{\mathbf{S C F}}+\mathbf{Z P E}$ & $\begin{array}{c}\mathbf{E}_{\mathbf{S C F}}+\mathbf{Z P E}+ \\
\mathbf{E}_{\text {int }}\end{array}$ & $\begin{array}{c}\mathbf{E}_{\mathbf{S C F}}+\mathbf{Z P E}+ \\
\mathbf{E}_{\text {int }}-\mathbf{T S}\end{array}$ & $\begin{array}{c}\mathbf{E}_{\mathbf{S C F}}+\mathbf{Z P E}+ \\
\mathbf{E}_{\text {int }}-\mathbf{T S}+\mathbf{G}_{\text {Sol }}\end{array}$ \\
\hline 1 & 0.000 & 0.000 & 0.000 & 0.000 & 0.000 \\
\hline $1-\mathrm{C}_{2 \mathrm{~V}}$ & 2.354 & 1.303 & 0.014 & 1.303 & 2.343 \\
\hline $1-\mathrm{C}_{\mathrm{S}} \mathrm{TS}$ & 10.947 & 10.536 & 10.088 & 10.377 & 8.907 \\
\hline 2 & 0.000 & 0.000 & 0.000 & 0.000 & 0.000 \\
\hline $2-\mathrm{C}_{2 \mathrm{~V}}$ & 0.892 & 0.267 & -0.610 & 0.303 & 0.493 \\
\hline $2-\mathrm{C}_{\mathrm{S}} \mathrm{TS}$ & 13.677 & 13.193 & 12.816 & 12.523 & 10.283 \\
\hline 3 & 0.000 & 0.000 & 0.000 & 0.000 & 0.000 \\
\hline $3-\mathrm{C}_{\mathrm{S}} \mathrm{TS}$ & 21.598 & 20.634 & 19.832 & 19.412 & 17.082
\end{tabular}

All energies are given in $\mathrm{kCal} / \mathrm{mol}$ at $298.15 \mathrm{~K}$

\begin{tabular}{l|r|r|r|r}
\multicolumn{1}{c|}{ Species } & \multicolumn{1}{c|}{$\mathbf{E}_{\mathbf{S C F}}$} & $\mathbf{E}_{\mathbf{S C F}}+\mathbf{Z P E}$ & $\mathbf{E}_{\mathbf{S C F}}+\mathbf{Z P E}+\mathbf{E}_{\text {int }}$ & $\mathbf{E}_{\mathbf{S C F}}+\mathbf{Z P E}+\mathbf{E}_{\text {int }}$ \\
& & & & $-\mathbf{T S}$ \\
\hline 1 & 0.000 & 0.000 & 0.000 & 0.000 \\
\hline $1-\mathrm{C}_{2 \mathrm{~V}}$ & 2.354 & 1.303 & 0.098 & 0.688 \\
\hline $1-\mathrm{C}_{\mathrm{S}} \mathrm{TS}$ & 10.947 & 10.536 & 10.094 & 10.237 \\
\hline 2 & 0.000 & 0.000 & 0.000 & 0.000 \\
\hline $2-\mathrm{C}_{2 \mathrm{~V}}$ & 0.892 & 0.267 & -0.446 & -0.099 \\
\hline $2-\mathrm{C}_{\mathrm{S}} \mathrm{TS}$ & 13.677 & 13.193 & 12.771 & 12.659 \\
\hline 3 & 0.000 & 0.000 & 0.000 & 0.000 \\
\hline $3-\mathrm{C}_{\mathrm{S}} \mathrm{TS}$ & 21.598 & 20.634 & 19.739 & 19.596
\end{tabular}

All energies are given in $\mathrm{kCal} / \mathrm{mol}$ at $150 \mathrm{~K}$ 
Hydride region of the variable temperature ${ }^{1} \mathrm{H}$ NMR spectra of a partially deuterated sample of $1 \mathrm{CF}_{3} \mathrm{COO}\left(500 \mathrm{MHz}, \mathrm{CDF}_{3} / \mathrm{CDF}_{2} \mathrm{Cl}\right)$

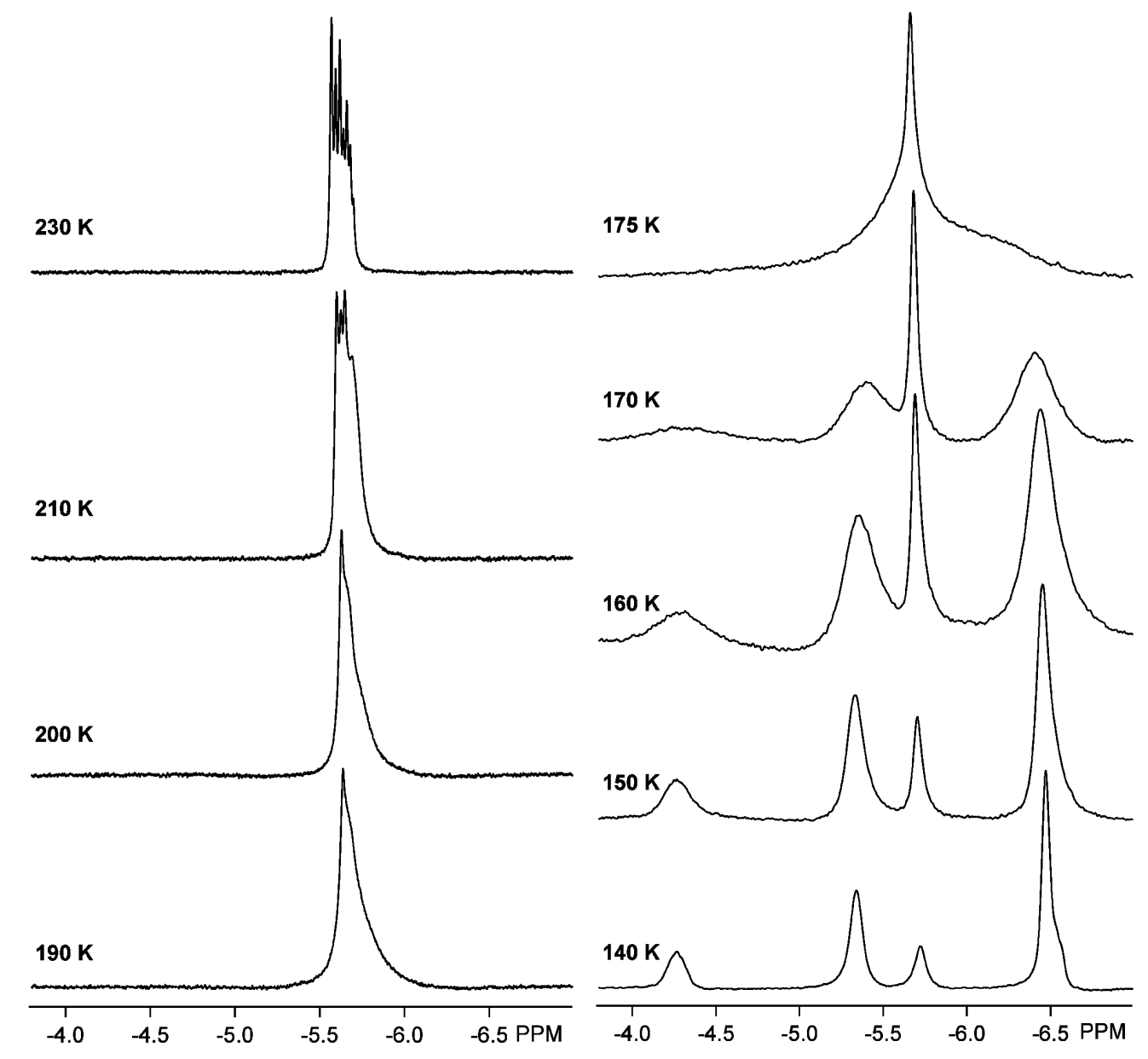


Hydride region of the variable temperature ${ }^{1} \mathrm{H}$ NMR spectra of a partially deuterated sample of $1 \mathrm{CF}_{3} \mathrm{COO}\left(750 \mathrm{MHz}, \mathrm{CD}_{2} \mathrm{Cl}_{2}\right)$
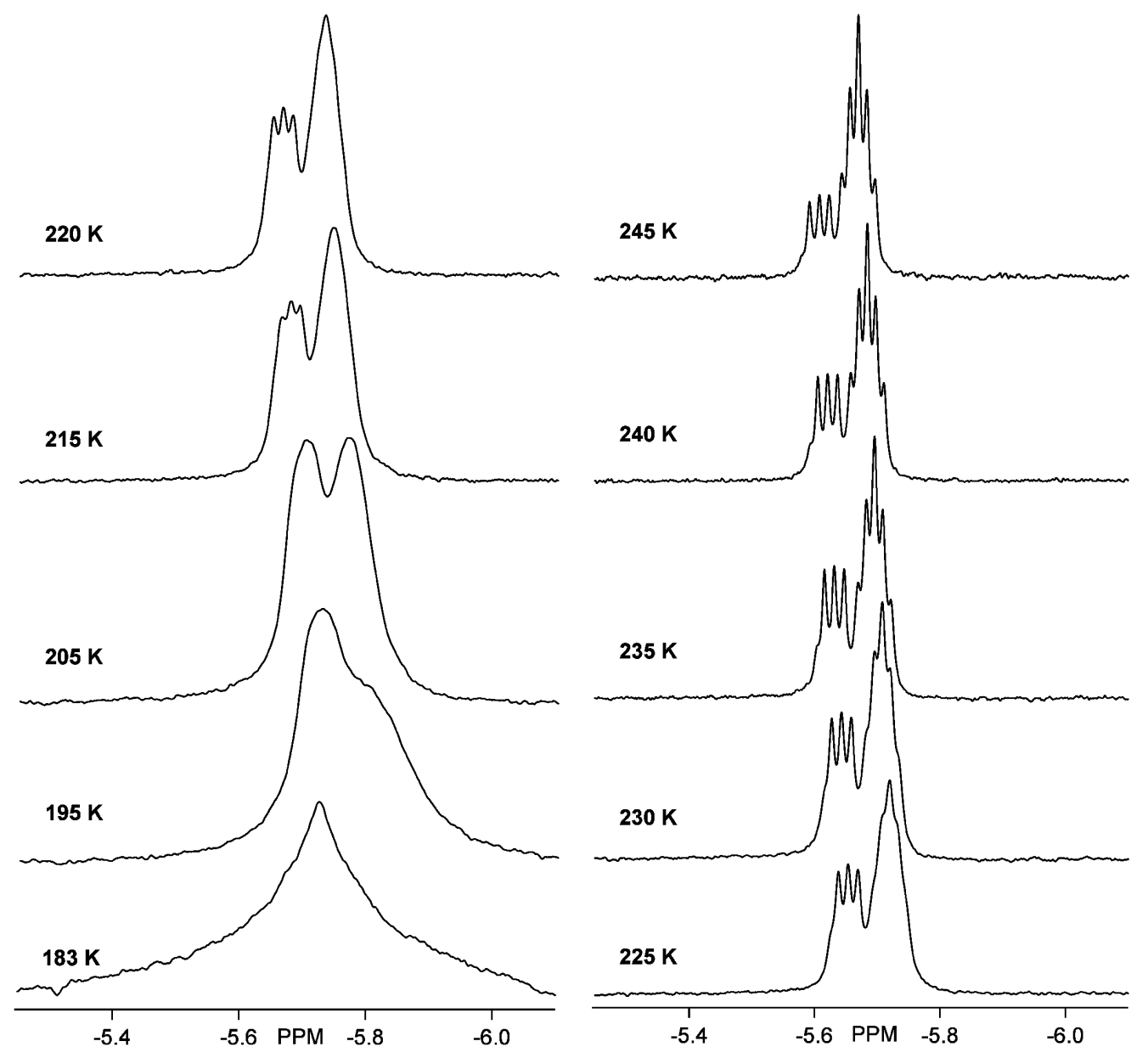
Plot of chemical shift and of the hydride resonance in 1, 1-d and 1-d as a function of temperature.

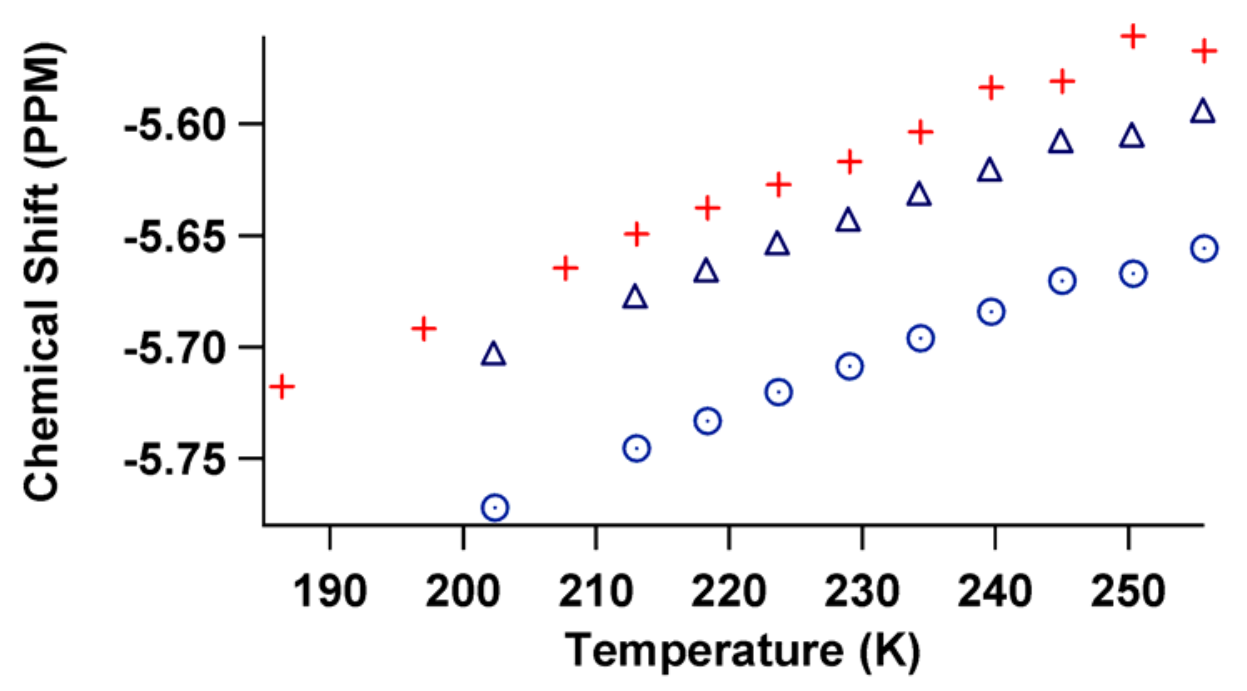

Plot of $\mathrm{H}-\mathrm{D}$ coupling in $1-d_{1}$ and $1-d_{2}$ as a function of temperature.

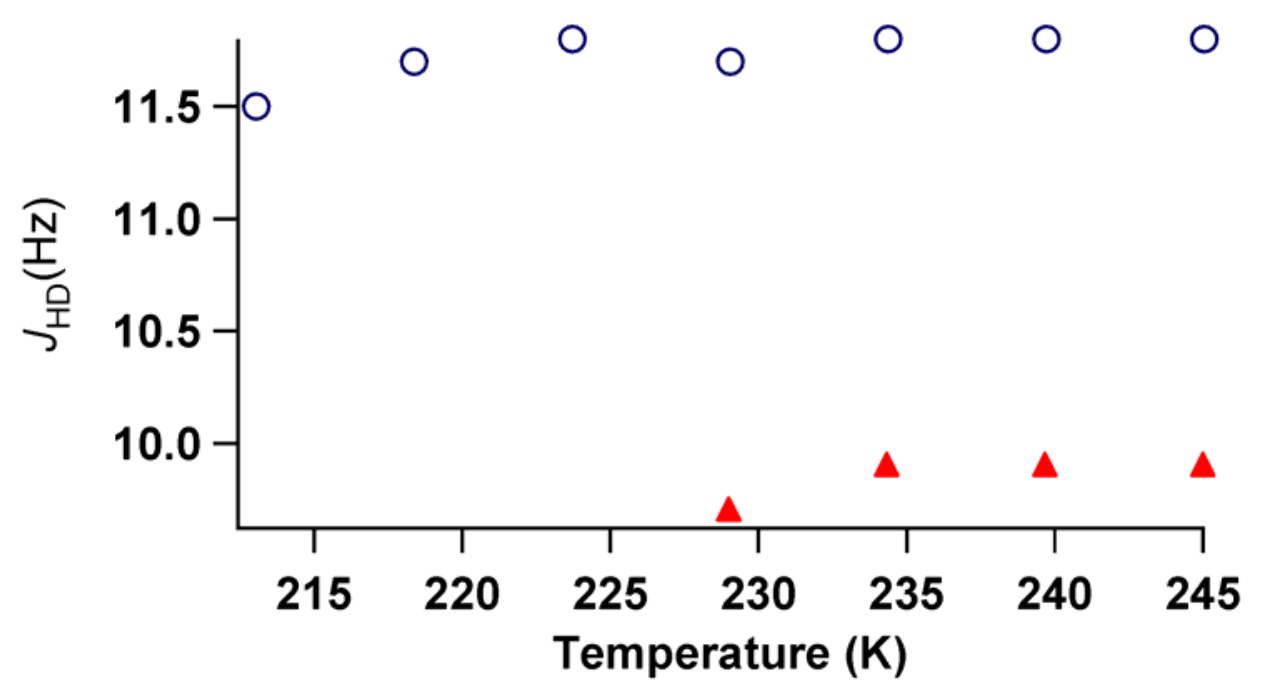

